\title{
Can augmented renal clearance be detected using estimators of glomerular filtration rate?
}

\author{
Matthias Gijsen ${ }^{1 *}$ D, Alexander Wilmer ${ }^{2}$, Geert Meyfroidt ${ }^{2}$, Joost Wauters ${ }^{2}$ and Isabel Spriet $^{1}$
}

Keywords: Augmented renal clearance, Intensive care unit, Creatinine clearance, Glomerular filtration rate

Estimators of glomerular filtration rate (GFR) have been shown to be flawed in critically ill patients, especially for augmented renal clearance (ARC), commonly defined as a measured urinary creatinine clearance $(\mathrm{CrCl}) \geq 130 \mathrm{ml} /$ $\min / 1.73 \mathrm{~m}^{2}$ [1]. Therefore, measuring $\mathrm{CrCl}$ should be performed in daily practice on the intensive care unit (ICU). However, many ICUs still rely on estimating formulae to monitor GFR [1, 2]. As estimators underestimate measured $\mathrm{CrCl}$ in ARC patients, ARC might remain unrecognized and lead to subtherapeutic plasma levels of drugs with predominant renal clearance [3]. Therefore, the aim of this study is to define the most precise GFR estimator, which can then be used to detect ARC when measured $\mathrm{CrCl}$ is unavailable.

We performed a multicenter retrospective registrybased [4] cohort study in adult ICUs from 3 tertiary university-affiliated hospitals in Belgium (Leuven, Ghent, Antwerp). All consecutive patients admitted between
January 2013 and December 2015 were screened for eligibility. All patients $\geq 18$ years old and having at least one measured 24-h urinary $\mathrm{CrCl}\left(\mathrm{CrCl}_{24 \mathrm{~h}}\right)$ were included. Agreement between $\mathrm{CrCl}_{24 \mathrm{~h}}$ and formulae estimating renal function, i.e., Cockcroft-Gault (CG), Chronic Kidney Disease Epidemiology Collaboration (CKD-EPI), and Modification of Diet in Renal Disease Study (MDRD), was evaluated on all included ICU days. For the estimator with the best precision, a cut-off for ARC with optimal specificity and sensitivity was identified, by calculating the Youden index [5]. Predictions for ARC using the cut-off value were compared to the actual presence of ARC based on the $\mathrm{CrCl}_{24 \mathrm{~h}}$. Cut-off values with either very high sensitivity (>95\%) or specificity (> 95\%) were also identified. Finally, the performance of these cut-offs was evaluated in an external single-center (Leuven, January 2016-December 2016) validation set by receiver-operating characteristics (ROC) curve ana-

\footnotetext{
* Correspondence: matthias.gijsen@uzleuven.be

Trial registration: The study was registered at ClinicalTrials.gov, NCT03954275. 'Pharmacy Department, Department of Pharmaceutical and Pharmacological Sciences, University Hospitals Leuven and Clinical Pharmacology and Pharmacotherapy, KU Leuven, Leuven, Belgium

Full list of author information is available at the end of the article
} 
Table 1 Agreement analysis between $\mathrm{CrCl}_{24 \mathrm{~h}}$ and formulae estimating renal function

\begin{tabular}{|c|c|c|c|}
\hline & All ICU days $(n=51,604)$ & $\mathrm{CrCl}_{24 \mathrm{~h}}<130 \mathrm{ml} / \mathrm{min} / 1.73 \mathrm{~m}^{2}(n=41,290)$ & $\mathrm{CrCl}_{24 \mathrm{~h}} \geq 130 \mathrm{ml} / \mathrm{min} / 1.73 \mathrm{~m}^{2}(n=10,314)$ \\
\hline \multicolumn{4}{|c|}{ Median (IQR) $\left(\mathrm{ml} / \mathrm{min} / 1.73 \mathrm{~m}^{2}\right)$} \\
\hline $\mathrm{CrCl} 24 \mathrm{~h}$ & $73(37 ; 118)$ & $58(30 ; 88)$ & $166(145 ; 200)$ \\
\hline $\mathrm{CrCl}_{\mathrm{CG}}$ & $83(50 ; 127)$ & $70(43 ; 103)$ & $145(116 ; 183)$ \\
\hline eGFR $\mathrm{MDRD}_{\mathrm{M}}$ & $87(50 ; 130)$ & $72(42 ; 109)$ & $143(115 ; 185)$ \\
\hline eGFR $R_{C K D-E P I}$ & $88(51 ; 108)$ & $75(43 ; 99)$ & $116(104 ; 130)$ \\
\hline \multicolumn{4}{|c|}{ Correlation with $\mathrm{CrCl}_{24 \mathrm{~h}}=$ Spearman correlation coefficient } \\
\hline $\mathrm{CrCl}_{C G}$ & $0.63^{\circ}$ & $0.62^{\circ}$ & $0.18^{\circ}$ \\
\hline eGFR $\mathrm{MDRD}_{\text {, }}$ & $0.59^{\circ}$ & $0.60^{\circ}$ & $0.15^{\circ}$ \\
\hline eGFR $R_{C K D-E P I}$ & $0.69^{\circ}$ & $0.72^{\circ}$ & $0.19^{\circ}$ \\
\hline \multicolumn{4}{|c|}{ Mean bias $(95 \% \mathrm{Cl})=$ mean difference $\mathrm{CrCl}_{24 \mathrm{~h}}$ - estimator $\left(\mathrm{ml} / \mathrm{min} / 1.73 \mathrm{~m}^{2}\right)$} \\
\hline $\mathrm{CrCl}_{\mathrm{CG}}$ & $-11(-11 ;-10)$ & $-20(-20 ;-19)$ & $25(23 ; 27)$ \\
\hline eGFR $R_{M D R D}$ & $-14(-15 ;-14)$ & $-23(-23 ;-23)$ & $21(19 ; 23)$ \\
\hline eGFR $R_{C K D-E P I}$ & $3(3 ; 4)$ & $-12(-13 ;-12)$ & $66(64 ; 67)$ \\
\hline \multicolumn{4}{|c|}{ Precision $=\mathrm{SD}$ of the bias $\left(\mathrm{ml} / \mathrm{min} / 1.73 \mathrm{~m}^{2}\right)$} \\
\hline $\mathrm{CrCl}_{\mathrm{CG}}$ & 55 & 41 & 83 \\
\hline eGFR $R_{M D R D}$ & 61 & 46 & 94 \\
\hline eGFR $R_{C K D-E P I}$ & $48^{*}$ & $26^{*}$ & $62^{*}$ \\
\hline \multicolumn{4}{|c|}{ Accuracy $=$ percentage within $30 \%$ of $\mathrm{CrCl}_{24 \mathrm{~h}}$} \\
\hline $\mathrm{CrCl}_{\mathrm{CG}}$ & 47 & 45 & 58 \\
\hline eGFR $R_{M D R D}$ & 45 & 43 & 56 \\
\hline eGFR $R_{C K D-E P I}$ & 50 & 51 & 45 \\
\hline \multicolumn{4}{|c|}{$\begin{array}{l}n \text { number of ICU days; } \mathrm{CrCl}_{24 h} \text { Creatinine clearance measured by 24-h urine collection, corrected for body surface area; } I Q R \text { interquartile range; } C r C l C G \text { estimated } \\
\text { creatinine clearance by the Cockcroft - Gault formula, corrected for body surface area; } e G F R_{M D R D} \text { estimated glomerular filtration rate by the 4-variable Modification } \\
\text { of Diet in Renal Disease formula; } e G F R R_{C K D-E P I} \text { estimated glomerular filtration rate by the Chronic Kidney Disease Epidemiology Collaboration formula; } S D \text { standard } \\
\text { deviation; } C l \text { confidence interval } \\
{ }^{o} p<0,001 \\
{ }^{*} \text { Best performing }\end{array}$} \\
\hline
\end{tabular}

lysis, using 2000 bootstrap replicates. The same inclusion and exclusion criteria as described above were applied.

A total of 51,604 ICU days were included to define a cut-off. Agreement analysis between $\mathrm{CrCl}_{24 \mathrm{~h}}$, the clinical reference, and the formulae estimating renal function is shown in Table 1. None of the estimators were precise (i.e., standard deviation of the mean bias was large for all estimators), with the CKD-EPI formula performing best over the whole $\mathrm{CrCl}_{24 \mathrm{~h}}$ range, and for ARC specifically, as illustrated in Fig. 1a. Hence, the CKD-EPI formula was selected for further analysis. In the validation set, 10,503 ICU days were included. For the CKD-EPI formula, the optimal cut-off for ARC was $96.5 \mathrm{ml} / \mathrm{min} /$ $1.73 \mathrm{~m}^{2}$. This cut-off showed a sensitivity of $86.6 \%$ [85; 88.1] and a specificity of $71 \%$ [70;71.9]. The cut-off values with very high sensitivity and specificity were $87.3 \mathrm{ml} / \mathrm{min} / 1.73 \mathrm{~m}^{2}$ (sens, $95.8 \%$ [95;96.7]; spec, $57.6 \%$ [56.6;58.7]) and $125.2 \mathrm{ml} / \mathrm{min} / 1.73 \mathrm{~m}^{2}$ (sens, 31.4\% [29.4; 33.5]; spec, $95.2 \%$ [94.7;95.6]), respectively. The ROC curve analysis including the cut-off values is shown in Fig. 1b. Evaluating the optimal cut-off in the validation set, we found that the proportion of accurate predictions for ARC decreased during the first 2 weeks of ICU stay. The is due to an increased false positive rate (Day-1, 16\%; Day-14, 49\%).

Overall, there was poor agreement between $\mathrm{CrCl}_{24 \mathrm{~h}}$ and GFR estimators, confirming previous literature [1]. However, the CKD-EPI formula, which is the "least worse" alternative to $\mathrm{CrCl}_{24 \mathrm{~h}}$, provided a cut-off with reasonable performance to detect ARC. Depending on the clinical context, this cut-off can be adapted to increase sensitivity or specificity. When applying this cutoff, the user should note that the accuracy decreases over time during the first 2 weeks of ICU stay. Hence, its largest benefit lies in the beginning of ICU stay. The presented CKP-EPI cut-off can be used to guide upfront increased antimicrobial dosing in patients presenting with ARC early upon ICU admission, when $\mathrm{CrCl}_{24 \mathrm{~h}}$ is not available. 


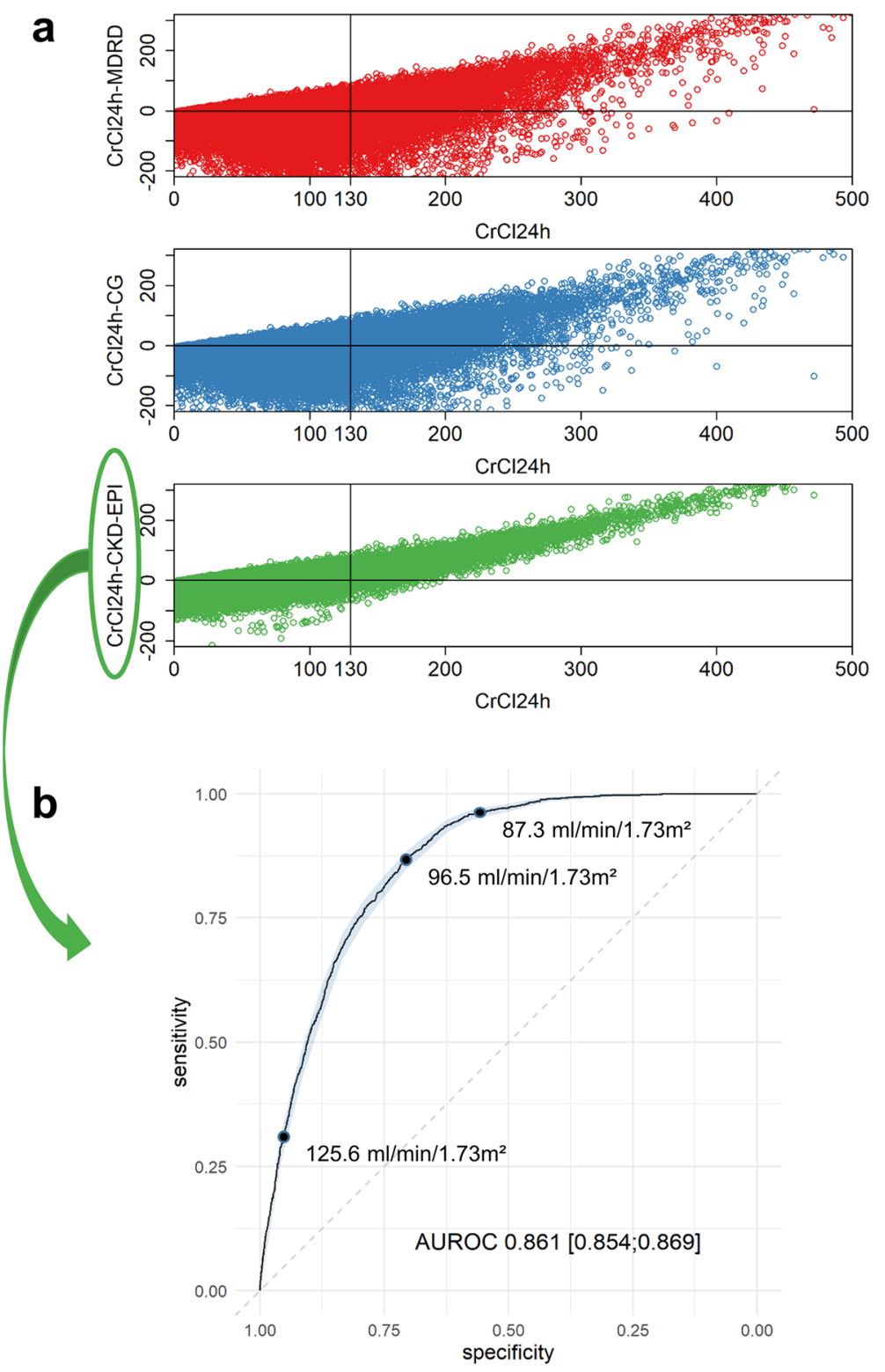

Fig. 1 a Bias in function of $\mathrm{CrCl}_{24 \mathrm{~h}}$ for the three formulae estimating renal function. Top: MDRD $\left.(\mathrm{ml} / \mathrm{min} / 1.73 \mathrm{~m})^{2}\right)$; mid: CG corrected for a body surface area of $1.73 \mathrm{~m}^{2}\left(\mathrm{ml} / \mathrm{min} / 1.73 \mathrm{~m}^{2}\right)$; bottom: CKD-EPI $\left(\mathrm{ml} / \mathrm{min} / 1.73 \mathrm{~m}^{2}\right)$. $\mathbf{b}$ Receiver operating characteristics curve analysis for the CKD-EPI formula. The shaded area represents the $95 \%$ confidence intervals. The dots represent the cut-off values for optimized sensitivity and specificity, very high (>95\%) sensitivity and very high (>95\%) specificity

\section{Abbreviations}

GFR: Glomerular filtration rate; ARC: Augmented renal clearance; $\mathrm{CrCl}$ : Creatinine clearance; ICU: Intensive care unit; $\mathrm{CrCl}_{24 \mathrm{~h}}$ : Measured 24-h urinary creatinine clearance; CG: Cockcroft-Gault formula; CKD-EPI: Chronic Kidney Disease Epidemiology Collaboration formula; MDRD: Modification of Diet in Renal Disease Study formula

\section{Acknowledgements}

The authors would like to thank the members of the M@tric research group for helpfully providing the M@tric database. The authors would also like to thank Astrid Eggerickx for her contribution to the analysis of the multicenter cohort.

\section{Authors' contributions}

MG, JW, and IS designed the study. GM and JW revised the study protocol. MG, AW, GM, and IS contributed to the data collection. MG, AW, JW, and IS were responsible for the analysis and interpretation of the data. MG and IS wrote the draft and all co-authors critically revised the manuscript and approved the final version for publication.

\section{Funding}

The Flemish Interuniversitary Intensive Care Database Project, later renamed M@tric, was funded by the Flemish government via the Hercules program of the Research Foundation, Flanders (FWO) (AUGE/09/022). GM receives 
funding from the FWO as senior clinical investigator $(1843118 \mathrm{~N})$, and project funding from the KU Leuven (C24/17/072). JW receives funding from FWO as senior clinical investigator (1833317 N). IS receives funding from the Clinical Research Fund of the University Hospitals Leuven, and project funding from the KU Leuven (C24/16/039).

\section{Availability of data and materials}

The datasets used and/or analyzed during the current study are available from the corresponding author upon reasonable request.

\section{Ethics approval and consent to participate}

M@tric data-collection has ethical committee approval from the University Hospitals Ghent, where the database is being hosted (PA 2009/006). Research on the M@tric database requires ethical committee approval of the ethical committee of one of the contributing centers which then independently acts as central ethical committee.

Approval for the present study was obtained from the ethical committee of the University Hospitals Leuven (\$61364) for the use of the M@tric dataset, as well as the retrospective Leuven dataset. This research has been performed in accordance with the ethical standards laid down in the 1964 Declaration of Helsinki and its later amendments.

\section{Consent for publication}

Not applicable.

\section{Competing interests}

The authors declare that they have no competing interests.

\section{Author details}

${ }^{1}$ Pharmacy Department, Department of Pharmaceutical and Pharmacological Sciences, University Hospitals Leuven and Clinical Pharmacology and Pharmacotherapy, KU Leuven, Leuven, Belgium. ${ }^{2}$ Clinical Division and Laboratory of Intensive Care Medicine, University Hospitals Leuven and Academic Department of Cellular and Molecular Medicine, KU Leuven, Leuven, Belgium.

Received: 8 May 2020 Accepted: 3 June 2020

Published online: 18 June 2020

\section{References}

1. Bilbao-Meseguer I, Rodriguez-Gascon A, Barrasa H, Isla A, Solinis MA Augmented renal clearance in critically ill patients: a systematic review. Clin Pharmacokinet. 2018;57(9):1107-21.

2. Ruiz S, Minville V, Asehnoune K, Virtos M, Georges B, Fourcade O, et al. Screening of patients with augmented renal clearance in ICU: taking into account the CKD-EPI equation, the age, and the cause of admission. Ann Intensive Care. 2015;5(1):49.

3. Udy AA, Roberts JA, Lipman J. Clinical implications of antibiotic pharmacokinetic principles in the critically ill. Intensive Care Med. 2013; 39(12):2070-82.

4. M@tric project [Available from: https://www.matric.be/]. Accessed 4 Nov 2019.

5. Fluss R, Faraggi $D$, Reiser B. Estimation of the Youden index and its associated cutoff point. Biom J. 2005;47(4):458-72.

\section{Publisher's Note}

Springer Nature remains neutral with regard to jurisdictional claims in published maps and institutional affiliations. 